\title{
Novel botanical drug DA-9803 prevents deficits in Alzheimer's mouse models
}

\author{
Guillaume J. Pagnier ${ }^{1 \dagger}$, Ksenia V. Kastanenka ${ }^{1 \dagger}$, Miwon Sohn², Sangzin $\mathrm{Choi}^{2}$, Song-hyen Choi ${ }^{2}$, HyeYeon Soh ${ }^{2}$ \\ and Brian J. Bacskai ${ }^{1^{*}}$
}

\begin{abstract}
Background: Alzheimer's disease (AD) is a neurodegenerative disorder characterized by deposition of amyloid plaques and disruption of neural circuitry, leading to cognitive decline. Animal models of AD deposit senile plaques and exhibit structural and functional deficits in neurons and neural networks. An effective treatment would prevent or restore these deficits, including calcium dyshomeostasis observed with in-vivo imaging.
\end{abstract}

Methods: We examined the effects of DA-9803, a multimodal botanical drug, in 5XFAD and APP/PS1 transgenic mice which underwent daily oral treatment with 30 or 100 mg/kg DA-9803 or vehicle alone. Behavioral testing and longitudinal imaging of amyloid deposits and intracellular calcium in neurons with multiphoton microscopy was performed.

Results: Chronic administration of DA-9803 restored behavioral deficits in 5XFAD mice and reduced amyloid- $\beta$ levels. DA9803 also prevented progressive amyloid plaque deposition in APP/PS1 mice. Elevated calcium, detected in a subset of neurons before the treatment, was restored and served as a functional indicator of treatment efficacy in addition to the behavioral readout. In contrast, mice treated with vehicle alone continued to progressively accumulate amyloid plaques and calcium overload.

Conclusions: In summary, treatment with DA-9803 prevented structural and functional outcome measures in mouse models of AD. Thus, DA-9803 shows promise as a novel therapeutic approach for Alzheimer's disease.

Keywords: Alzheimer's disease, Multiphoton microscopy, Therapeutic, In vivo, Calcium imaging

\section{Background}

Alzheimer's disease (AD) is a progressive neurodegenerative disorder and the most prevalent form of dementia [1]. AD is defined by the presence of amyloid plaques and neurofibrillary tangles in the brain [2]. Overwhelming evidence suggests that the aggregation of amyloidbeta $(A \beta)$ initiates a cascade of events that lead to formation of amyloid plaques and neurofibrillary tangles that culminate in loss of neurons [3]. Transgenic mouse models that overexpress human amyloid precursor protein (APP) develop $A \beta$-related pathologies such as amyloid plaque deposition similar to those in AD patients [4-6]. Additionally, as these mice age, their cortical neurons exhibit alterations in calcium homeostasis,

\footnotetext{
* Correspondence: bbacskai@mgh.harvard.edu

${ }^{\dagger}$ Equal contributors

'Department of Neurology, MassGeneral Institute of Neurodegenerative Diseases, Massachusetts General Hospital and Harvard Medical School, 114 Sixteenth St., Charlestown, MA 02129, USA

Full list of author information is available at the end of the article
}

supporting the calcium hypothesis of AD [7-11]. Approximately $20 \%$ of neuronal processes exhibit aberrant calcium homeostasis leading to elevated levels of resting calcium, or calcium overload, in APP/PS1 mice aged 810 months with substantial amyloid pathology, and this is not related to the presenilin gene [11]. Calcium overload also correlates with disrupted neuronal structure and function [11], so restoring calcium homeostasis could serve as an indirect functional indicator of treatment efficacy. Furthermore, it is well documented that neuroinflammation is central to the progression of the disease, although it is not clear whether it is a cause or a consequence of the disease. An increased number of reactive microglia and astrocytes are concentrated in close proximity to amyloid plaques in humans and mouse models [12-14]. Thus, an effective AD treatment would halt $A \beta$ deposition, restore intraneuronal calcium to control levels, and improve the ability of neuroinflammatory cascades to respond to pathology. 
DA-9803 is a multimodal, botanical therapeutic currently in preclinical development by Dong-A ST that shows nootropic promise. It is a well-controlled proprietary extract from Morus alba $L$. and the surface layer of Poria cocos that contains multiple major and minor active ingredients. Its pharmacokinetics and mechanism of action are currently under study. The extract is carefully and reproducibly prepared similar to other natural product extracts [15]. Thus, the extract, with no obvious indications of toxicity, is a potential drug candidate for the treatment and prevention of $\mathrm{AD}$.

This work tested the ability of DA-9803 to reverse the structural and functional deficits in two separate $A D$ mouse models. 5XFAD mice show behavioral deficits as they age, which was restored with DA-9803 treatment in parallel with a decrease in $A \beta$ levels measured with ELISA. Multiphoton imaging in a separate cohort of APP/PS1 mice treated with DA-9803 was used to monitor the dynamics of plaque deposition [16] and neuronal calcium levels in the cortex during a 2-month treatment period $[16,17]$. Treating young APP/PS1 mice with DA9803 halted $\mathrm{A} \beta$ plaque deposition and decreased the number of neuronal processes exhibiting calcium overload. These results suggest that DA-9803 affects $A \beta$ aggregation as well as functional outcome measures in mouse models, and warrants further investigation of this promising therapeutic.

\section{Methods}

\section{Animals and surgery}

Five-month-old 5XFAD transgenic mice (five males/group) were used for the behavioral analyses. 5XFAD transgenic mice overexpress both mutant human APP(695) with the Swedish (K670N, M671L), Florida (I716V), and London (V717I) mutations as well as human PS1 harboring two FAD mutations, M146L and L286V [6]. The behavioral experiments were approved by Institutional Animal Care and Use Committee of Dong-A ST and conducted according to the IACUC guidelines.

Transgenic APPSwe/PS1dE9 mice were used for longitudinal imaging studies (eight males, five females). APP/ PS1 mice overexpress the Swedish mutation in the APP gene, as well as the delta E9 mutation in the PS1 gene [5]. The studies were conducted in compliance with Massachusetts General Hospital Animal Care and Use Committee and NIH guidelines for the use of experimental animals.

At 5 months of age, the APP/PS1 animals underwent intracortical virus injections followed by craniotomies as described previously $[18,19]$. Briefly, each animal was anesthetized with $2 \%$ isoflurane and placed in a sterotaxic apparatus. Body temperature was maintained with a heating pad throughout the course of anesthesia. The skin over the skull was disinfected with betadine and isopropyl alcohol, and then cut to reveal the skull. Burr holes were drilled through the skull in each hemisphere with the following coordinates: A-P $-3 \mathrm{~mm}, \mathrm{M}-\mathrm{L}-1 \mathrm{~mm}, \mathrm{D}-\mathrm{V}-$ $0.8 \mathrm{~mm}$ with respect to the bregma. Using a Hamilton syringe, $1.5 \mu \mathrm{l}$ of AAV8 Yellow Cameleon 3.6 (YC3.6) (U. Penn Viral Core) was injected $0.8 \mathrm{~mm}$ below the dura in both burr holes at a rate of $130 \mathrm{nl} / \mathrm{min}$ to target excitatory neurons in the somatosensory cortex. YC3.6 is a genetically encoded ratiometric calcium indicator that reports calcium concentrations quantitatively $[11,20]$. A cranial window was then installed by first performing a round craniotomy over the somatosensory cortex. The area over the brain was covered with an 8-mm glass coverslip and fixed with a mixture of crazy glue and dental cement [18, $19,21]$. Each animal remained on a heating pad while recovering from anesthesia. To allow for YC3.6 expression and to limit an inflammatory response associated with the craniotomy interfering with multiphoton imaging, DA9803 treatment commenced 4 weeks after virus injections and installation of cranial windows. Mice were 6 months old at this point.

\section{Chronic DA-9803 treatment}

Each 5XFAD mouse was assigned randomly to either the vehicle or the DA-9803 treatment condition for the behavioral studies. Then $200 \mu \mathrm{l}$ of the vehicle or the DA9803 solution was administered via oral gavage daily. DA-9803 is a botanical drug that can be dissolved in saline solution. The vehicle consisted of phosphate buffered saline (PBS) and hydroxypropyl methyl cellulose (HPMC). The drug treatment was composed of the vehicle solution as well as DA-9803. Animals in the drug condition received doses of either 30 or $100 \mathrm{mg} / \mathrm{kg}$ DA9803. After 4 weeks of daily gavage treatment, the 5XFAD mice were subjected to the Y-maze and the passive avoidance tests. Animals were subsequently euthanized and their brains processed for biochemistry.

For the longitudinal imaging experiments, APP/PS1 animals were assigned randomly to either the vehicle or the DA-9803 treatment condition. Then $300 \mu$ of the vehicle or the DA-9803 solution was administered by oral gavage daily. The vehicle consisted of PBS and HPMC. DA-9803 was added to the vehicle formulation for administration to the treated mice. Animals in the drug condition received a dose of $100 \mathrm{mg} / \mathrm{kg}$ DA-9803. Daily gavage treatment commenced after the first multiphoton imaging session. Animals were subsequently imaged 2, 4, and 8 weeks after the first imaging session. Daily gavage treatment was continued until the last imaging session, after which the animals were perfused transcardially with PBS, and their brains extracted and processed for immunohistochemistry. Of the 13 animals that were imaged, seven were treated with the vehicle and six were treated with DA-9803. 


\section{Multiphoton imaging and data acquisition}

The day prior to the first imaging session, mice were injected intraperitoneally with $4 \mathrm{mg} / \mathrm{kg}$ methoxy-XO4 to label amyloid plaques [11, 22]. Texas Red-labeled dextran was injected via the retro-orbital sinus to provide a fluorescent angiogram. Prior to subsequent imaging sessions, animals were reinjected with methoxy-XO4 to label newly appeared amyloid plaques. Imaging of amyloid plaques and YC3.6-positive neurons was performed using an Olympus Fluoview 1000MPE mounted on an Olympus BX61WI upright microscope. A $25 \times$ water immersion objective $(\mathrm{NA}=1.05)$ was used for imaging. A mode-locked titanium:sapphire laser (MaiTai; SpectraPhysics, Fremont, CA, USA) generated two-photon fluorescence with either 800 or $860 \mathrm{~nm}$ excitation. Amyloid plaque pathology was imaged using $800 \mathrm{~nm}$ excitation at $1 \times$ zoom. YC3.6 was imaged using $860 \mathrm{~nm}$ excitation at $1 \times, 2 \times$, and $5 \times$ zoom. Laser power was kept below $30 \mathrm{~mW}$ to avoid phototoxicity.

After each imaging session, mice were allowed to recover from anesthesia on a heating pad. At the end of the last imaging session, mice were euthanized with $\mathrm{CO}_{2}$, perfused with $4 \%$ paraformaldehyde in PBS, and fixed with $4 \%$ paraformaldehyde and $15 \%$ glycerol cryoprotectant overnight. Brains were frozen in Optimal Cutting Temperature compound (OCT), cut into $20-\mu \mathrm{m}$ coronal sections on a cryostat, and mounted onto slides.

\section{Image processing and data analysis}

Image stacks acquired in vivo were analyzed using ImageJ software (http://rsbweb.nih.gov/ij/). Images were analyzed to determine amyloid plaque numbers, amyloid plaque burden, and resting calcium levels within individual neuronal processes (neurites). The same neurites were followed longitudinally to determine changes in calcium levels in each individual, identified neurite. Neurites that were not present in all four imaging sessions were excluded from analysis. For amyloid plaque analysis, each z-stack was processed into a maximum intensity projection. To determine the amyloid plaque number, amyloid plaques were counted manually in each projected image. To calculate the amyloid burden, each projected image was thresholded, segmented, and the percentage area occupied by amyloid was measured. Any signal from the amyloid lining the blood vessels, cerebral amyloid angiopathy, was excluded from analysis.

YC3.6 images were also analyzed using ImageJ. YC3.6 is a FRET probe, composed of a donor, cyan fluorescent protein (CFP), and an acceptor, yellow fluorescent protein (YFP) [20]. Intraneuronal concentrations were determined from the ratio of YFP to CFP. The higher the calcium concentration, the higher the ratio of YFP to CFP. The background for each channel was calculated by the mode of the intensities of the last slice of each volume and was subtracted from its respective channel. A median filter with a radius of 2 was applied and the fluorescence intensity of YFP was divided by CFP to create a ratio image. Neurites were identified manually and selected using the 'free hand' tool on ImageJ in the YFP images to minimize bias based on the calcium concentration. These neuronal regions of interest (ROIs) were then exported to the ratio images and the YFP/CFP ratios calculated. The relative change in YFP/CFP ratio $\left(\Delta R / R_{i}\right)$ was calculated by tracking the same neurites throughout all four imaging sessions. YFP/CFP ratios were converted to $\left[\mathrm{Ca}^{2+}\right]$ with standard equations using the in-situ $K_{\mathrm{d}}$ and Hill coefficient for YC3.6 determined previously [11]. Pseudocolored images were created using Matlab based on the calcium concentration using the empirical $R_{\min }$ and $R_{\max }$. The ratio values were used to determine the hue and saturation (color) and the brightness values were used to determine the value (intensity) in the pseudocolored images.

\section{Immunohistochemistry}

Twenty-micrometer transverse coronal sections of mouse brain underwent antigen retrieval in citrate buffer. The coronal sections were then permeabilized with Triton X-100, blocked with normal goat serum (NGS), and incubated with the various primary antibodies: $6 \mathrm{E} 10$ (monoclonal 6E10, 1:500; Covance), IBA1 (rabbit antiIba1, 1:2000; Wako), glial fibrillary acidic protein (GFAP) (mouse anti-GFAP, 1:200; Thermo Scientific), and MAP2 (mouse anti-MAP2, 1:100; Abcam) for 2 hours at room temperature. The coronal sections were then incubated with the respective secondary antibodies for 1 hour and mounted with ProLong Antifade reagent (Invitrogen).

\section{Postmortem image analysis}

To image amyloid plaque burden post mortem, images of entire cortical hemispheres or hippocampi were acquired using an inverted Zeiss microscope with a 10x objective. Images were analyzed using Image). MethoxyXO4-labeled plaques were thresholded and the amyloid burden was calculated as a percentage of the cortical (or hippocampal) area.

GFAP, 6E10, MAP2, and IBA1-stained slides were also imaged using an inverted Zeiss microscope at $20 x$ and $40 x$ zoom. The objective was either centered around a single plaque or positioned over an area lacking plaques. Microglia and astrocytes present in the field of view were counted manually. Microglial and astrocytic process length and cell body diameters were analyzed using ImageJ.

\section{Y-maze test}

The Y-maze consisted of three black, opaque, plastic arms $(5 \mathrm{~cm} \times 30 \mathrm{~cm} \times 12 \mathrm{~cm}) 120^{\circ}$ from each other. The 5XFAD mice were placed in the center and were allowed 
to explore all three arms. The number of arm entries and number of triads (a triad constituted subsequent entries into three separate arms) were recorded to calculate the percentage of alternation. An entry was defined as all four appendages entering a Y-maze arm. Alternation behavior was defined as the number of triads divided by the number of arm entries minus 2 and multiplied by 100.

\section{Passive avoidance test}

The passive avoidance chamber was divided into a white (light) and a black (dark) compartment (both $20 \mathrm{~cm} \times$ $20 \mathrm{~cm} \times 20 \mathrm{~cm}$ ). The light compartment contained a 50W electric lamp. The floor of the dark department contained a number of 2-mm stainless steel rods spaced $1 \mathrm{~cm}$ apart. Each mouse was placed in the light compartment. The door separating the two compartments was opened 10 seconds later. Once the mouse entered the dark compartment, the door closed and an electrical foot shock $(0.1 \mathrm{~mA} / 10 \mathrm{~g})$ was delivered through the grid floor for 3 seconds. Twenty-four hours after the training trial, mice were placed in the light chamber for testing. Latency was defined as the time it took for a mouse to enter the dark chamber after the door separating the two compartments opened.

\section{Amyloid-beta ELISAs}

Total (soluble and insoluble) $A \beta 40$ and $A \beta 42$ levels were detected using the $A \beta 40$ or $A \beta 42$ sandwich ELISA kits. Each 5XFAD cerebral cortex was homogenized in icecold buffer and centrifuged at $14,000 \times g$ for 30 minutes at $4{ }^{\circ} \mathrm{C}$ to isolate $\mathrm{A} \beta$. The resulting supernatants were used for the immunoassay. Standards and tissue samples were incubated in rabbit anti-mouse $A \beta 40$ or $A \beta 42$ polyclonal antibody-precoated 96-well immunoplates overnight at $4{ }^{\circ} \mathrm{C}$ (KMB3481 and KMB3441; Invitrogen). The plates were incubated with anti-rabbit IgG HRP for 30 minutes at room temperature. After washing, the plates were incubated with stabilized Chromogen for 30 minutes in the dark. Stop solution was then added to each well. The plates were read at $450 \mathrm{~nm}$ using a spectrophotometer (Spectramax microplate reader; Molecular Devices). Concentrations of $A \beta 40$ or $A \beta 42$ were determined in the sample solution using $A \beta 40$ or $A \beta 42$ standard calibration curves. The protein from the samples was normalized using the Bio-Rad assay for total protein determination.

\section{Statistics}

Statistical analyses were conducted in GraphPad 5.0. Data were expressed as mean \pm SEM. Datasets were checked for normality (Shapiro-Wilk normality test or Kolmogorov-Smirnov test) and appropriate statistical tests were used (parametric $t$ test or ANOVAs for normally distributed data, Mann-Whitney or KruskalWallis test for nonparametric data). $p<0.05$ was considered significant.

\section{Results}

One-month daily treatment with $30 \mathrm{mg} / \mathrm{kg}$ and $100 \mathrm{mg} /$ kg DA-9803 restores behavioral deficits and decreases $A \beta$ peptide levels in 5XFAD mice

5XFAD mice were used to determine the effect of DA9803 treatment on functional recovery as well as $A \beta 40$ and $A \beta 42$ levels in the cerebral cortex. Mice were treated via daily gavage with the vehicle, $30 \mathrm{mg} / \mathrm{kg}$ DA9803, or $100 \mathrm{mg} / \mathrm{kg}$ DA-9803. Animal behavior was monitored using the Y-maze and passive avoidance paradigms. Prior to treatment, 5XFAD mice, regardless of their assigned treatment condition, showed impairment as a function of decreased alternation behavior in the Ymaze, consistent with the literature (Fig. 1a, ANOVA followed by Student-Newman-Keuls method, $n=5$ mice/group) [6]. After 1 month of treatment, the $30 \mathrm{mg} /$ $\mathrm{kg}$-treated and $100 \mathrm{mg} / \mathrm{kg}$-treated mice showed improvement in alternation behavior between the arms of the Y-maze compared to the vehicle-treated 5XFAD mice (Fig. 1b, ANOVA followed by Student-NewmanKeuls method, $n=5$ mice/group). Moreover, animals in the $100 \mathrm{mg} / \mathrm{kg}$ condition recovered to the levels of wildtype controls (average of $71.7 \pm 4.0 \%$ and $74.0 \pm 6.2 \%$ respectively) (Fig. 1b). 5XFAD mice showed impairment in the passive avoidance task as well, spending $124.8 \pm$ 20.3 seconds in the light compartment before returning to the dark compartment when treated with vehicle compared to $300.0 \pm 0.0$ seconds for the wildtype mice (Fig. 1c, individual $t$ tests, $n=5$ mice/group). The $30 \mathrm{mg} / \mathrm{kg}$-treated and $100 \mathrm{mg} / \mathrm{kg}$-treated mice spent significantly longer periods of time in the light compartment compared to the vehicle-treated mice (189.6 \pm 41.1 seconds and $195.0 \pm 13.71$ seconds respectively compared to $124.8 \pm 20.3$ seconds), indicative of functional recovery (Fig. 1c). Thus, 1 -month $30 \mathrm{mg} / \mathrm{kg}$ and $100 \mathrm{mg} / \mathrm{kg}$ DA-9803 treatment improves the behavioral deficit in the Y-maze and the passive avoidance task in 5XFAD mice, suggesting that DA-9803 has beneficial effects in these animals.

To investigate the effect of DA-9803 treatment on $A \beta$, the $A \beta 40$ and $A \beta 42$ levels were measured using ELISAs in 5XFAD mice. $A \beta 40$ and $A \beta 42$ analyses showed similar trends. $A \beta 40$ and $A \beta 42$ levels were the lowest in wildtype mice $(134.6 \pm 60 \mathrm{pg} / \mathrm{mg}$ and $2.44 \pm 0.24 \mathrm{pg} / \mathrm{mg}$ respectively) (Fig. 1d, e). Vehicle-treated and $30 \mathrm{mg} / \mathrm{kg}$-treated mice had comparable levels of $A \beta 40$ and $A \beta 42$ after treatment (vehicle-treated mice: $546.4 \pm 48.8 \mathrm{pg} / \mathrm{mg}$ of $\mathrm{A} \beta 40$ and $50.7 \pm 2.1 \mathrm{pg} / \mathrm{mg}$ of $\mathrm{A} \beta 42 ; 30 \mathrm{mg} / \mathrm{kg}$-treated mice: $521.4 \pm 99.9 \mathrm{pg} / \mathrm{mg}$ of $\mathrm{A} \beta 40$ and $52.6 \pm 8.3 \mathrm{pg} / \mathrm{mg}$ of A 442 ) (Fig. 1d, e). The $100 \mathrm{mg} / \mathrm{kg}$ treatment significantly 

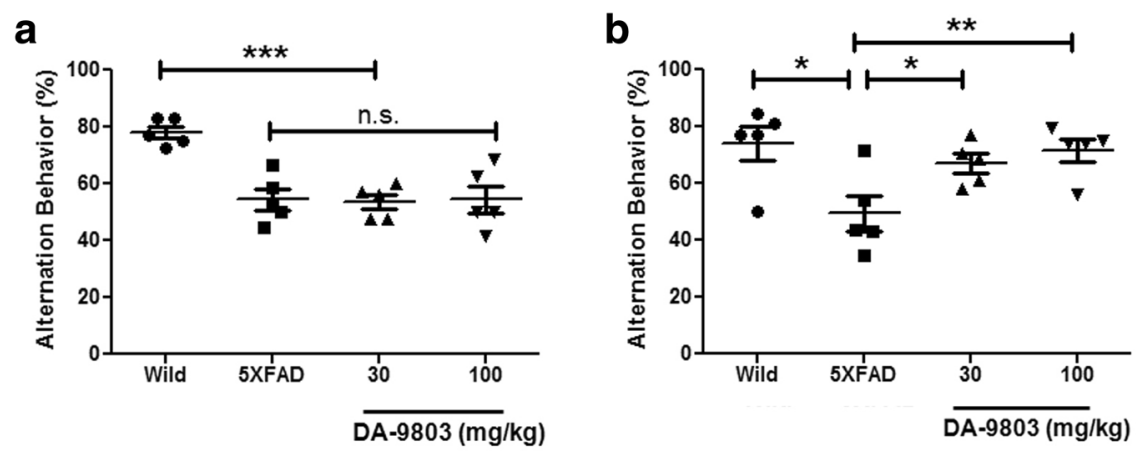

C
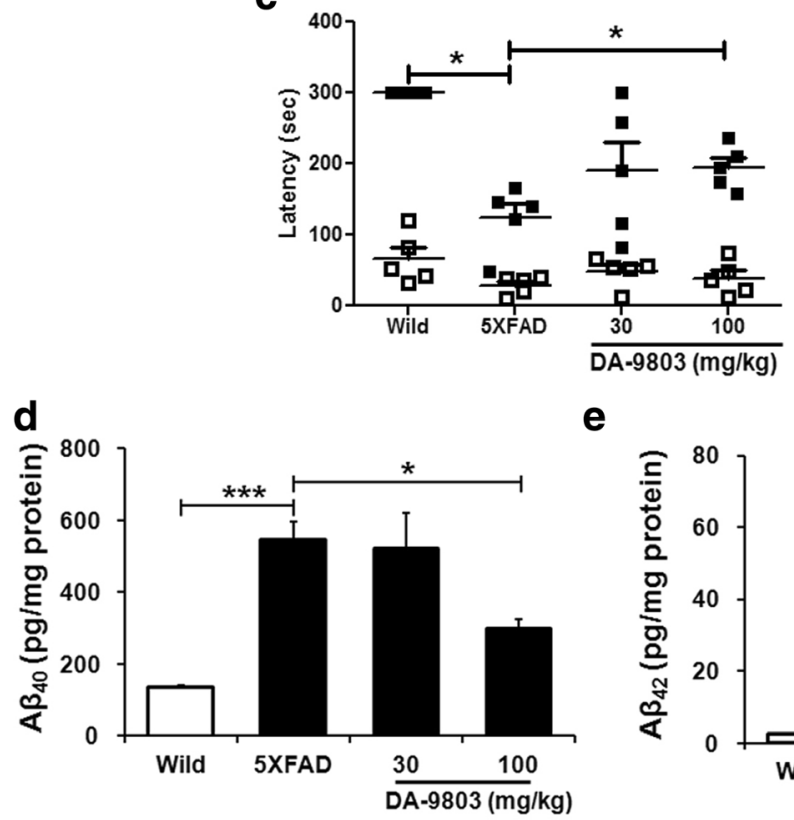

e

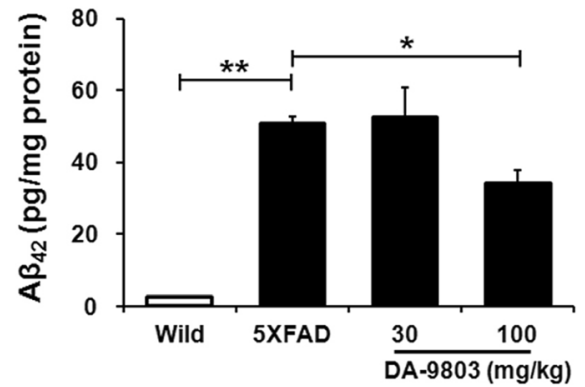

Fig. 1 Chronic treatment with DA-9803 restores behavioral deficits and decreases A 3 levels in cortices of 5XFAD mice. a, b Alternation behavior in a Y-maze before (a) and after (b) 4-week chronic DA-9803 treatment in 5-month-old 5XFAD mice ( $n=5$ mice/group). c Latency of entry into the dark compartment on a passive avoidance test after 4-week DA-9803 treatment ( $n=5$ mice/group). d, e Total A $\beta 40$ (d) and A 442 (e) levels in cortices of 5XFAD mice after 4-week DA-9803 treatment ( $n=5$ mice/group). Mean \pm SEM. ${ }^{*} p<0.05,{ }^{* *} p<0.01,{ }^{* * *} p<0.001$. n.s. not significant, $A \beta$ amyloid-beta

lowered the levels of $A \beta 40$ and $A \beta 42$ compared to the vehicle $(297.3 \pm 26.7 \mathrm{pg} / \mathrm{mg}$ of $A \beta 40$ and $34.1 \pm 3.9 \mathrm{pg} / \mathrm{mg}$ of A 342 , ANOVA followed by Newman-Keuls post-hoc test, $n=5$ cortices/group) (Fig. 1d, e). These data suggest that DA-9803 is reducing production or increasing clearance of $A \beta 40$ and $A \beta 42$ via a dose-dependent mechanism.

\section{Two-month daily treatment with DA-9803 halts plaque} deposition in-vivo in APP/PS1 mice

APP/PS1 mice were used to monitor the longitudinal effect of DA-9803 treatment on plaque deposition. Mice were treated via daily gavage with either the vehicle or $100 \mathrm{mg} / \mathrm{kg}$ DA-9803 for 2 months (Fig. 2a). Individual plaques were imaged over time with multiphoton microscopy (Fig. 2b-g). Angiograms were used as fiducial markers to find the plaques in the same locations over time (Fig. 2b, e). Mice in the vehicle and the drug conditions started with a similar number of plaques in the somatosensory cortex: vehicletreated mice exhibited $86 \pm 17$ plaques $/ \mathrm{mm}^{3}$ while DA9803-treated mice had $89 \pm 9$ plaques $/ \mathrm{mm}^{3}$ (mean \pm SEM) at baseline (Fig. 2h). Throughout the 2-month treatment period, animals treated with the vehicle exhibited an increase in the number of amyloid plaques accumulating to an average of $140 \pm 45$ plaques $/ \mathrm{mm}^{3}$ by the end of treatment, statistically different from baseline (mean $\pm \mathrm{SEM}$, Mann-Whitney test $p<0.001, n=33$ z-stacks in six mice treated with vehicle) (Fig. 2h). Conversely, animals treated with DA-9803 exhibited a decrease in amyloid plaque number and thus showed both prevention of plaque deposition and plaque clearance (average of $67 \pm 20$ plaques $/ \mathrm{mm}^{3}$ at the end of DA-9803 treatment, significantly different from baseline, Mann-Whitney test $p<0.01, n=30$ z-stacks in six 


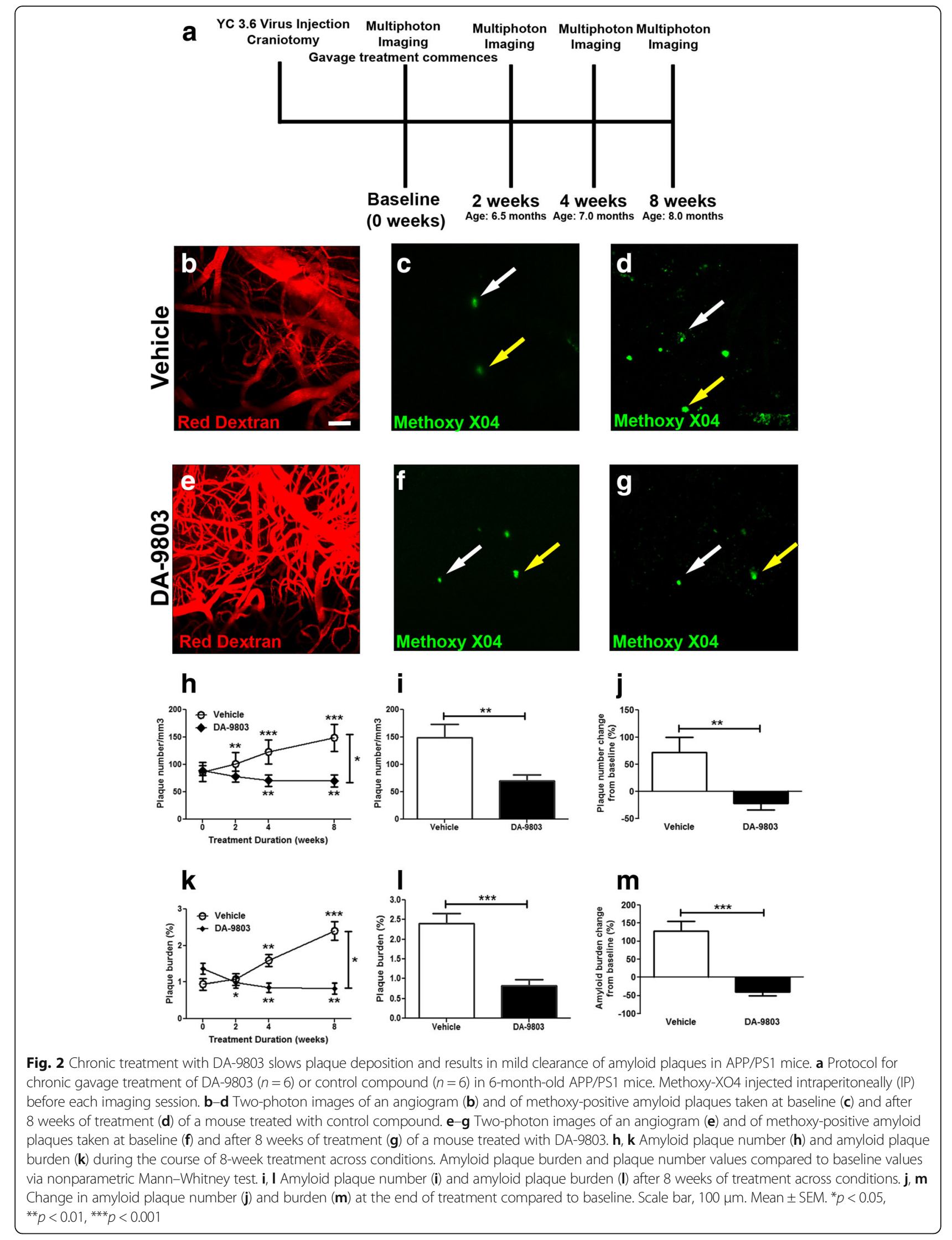


mice treated with DA-9803) (Fig. 2h). Thus, the rates of amyloid plaque deposition were different between the two conditions (simple linear regression $p<0.05$ ) (Fig. 2h). By the end of treatment, the number of amyloid plaques was significantly lower in DA-9803-treated compared to vehicletreated animals (Fig. 2i). Likewise, the amyloid burden, which was similar at the beginning of treatment, increased in the vehicle condition and decreased in the drug condition (Fig. 2k). Hence, amyloid plaque burden was significantly lower after the DA-9803 treatment compared to the vehicle (Mann-Whitney test $p<0.001, n=30 \mathrm{z}$-stacks in six mice treated with DA-9803, $n=33$ z-stacks in six mice treated with vehicle; Fig. 2l). Therefore, DA-9803-treated mice exhibited a $21.6 \pm 11.94 \%$ reduction in amyloid plaque number compared to baseline, while vehicle-treated mice presented a $72.13 \pm 27.85 \%$ increase (Fig. 2j). Similarly, amyloid plaque burden decreased by the end of the DA-9803 treatment compared to baseline, while increasing in the vehicle condition (Fig. 2m). Thus, daily oral treatment with DA-9803 halted plaque deposition and led to a reduction in amyloid plaque number and amyloid plaque burden in APP/PS1 mice over the 2-month period.

To confirm the in-vivo findings, immunohistochemistry with the anti- $\mathrm{A} \beta$ antibody $6 \mathrm{E} 10$ was performed on the 20 - $\mu \mathrm{m}$ thick coronal sections of brains isolated from animals that underwent amyloid imaging in vivo. Amyloid plaque burden was visualized ex vivo with the methoxy-XO4 signal that remained from in-vivo imaging as well as with 6E10. Methoxy-XO4 labeling colocalized with 6E10 immunostaining in vehicle-treated animals (Fig. $3 \mathrm{a}-\mathrm{c}$ ) and those treated with DA-9803 (Fig. 3d-f). Methoxy-XO4 labeled dense core plaques, while $6 \mathrm{E} 10$ immunolabeled the $\mathrm{A} \beta$ including and surrounding dense core plaques (Fig. 3c, f), similar to earlier reports [22, 23]. We analyzed 6E10labeled and methoxy-XO4-labeled amyloid burden in the cortices of mice treated with DA-9803 and the vehicle $(n=$ 19 cortical sections in six mice treated with DA-9803, $n=$ 20 cortical sections in six mice treated with vehicle). The amyloid burden detected with 6E10 in DA-9803-treated mice was significantly lower than the amyloid burden in vehicle-treated mice $(0.75 \pm 0.10 \%$ plaque load vs $1.4 \pm$ $0.28 \%$ plaque load, Mann-Whitney test $p<0.05$ ) (Fig. 3g). Likewise, the amyloid burden detected with methoxy-XO4 in DA-9803-treated mice was significantly lower than the amyloid burden in vehicle-treated mice in the cortex $(0.09$ $\pm 0.02 \%$ plaque load vs $0.22 \pm 0.04 \%$ plaque load, MannWhitney test $p<0.05$ ) (Fig. 3h) and the hippocampus $(0.03 \pm 0.01 \%$ plaque load vs $0.14 \pm 0.03 \%$ plaque load $)$
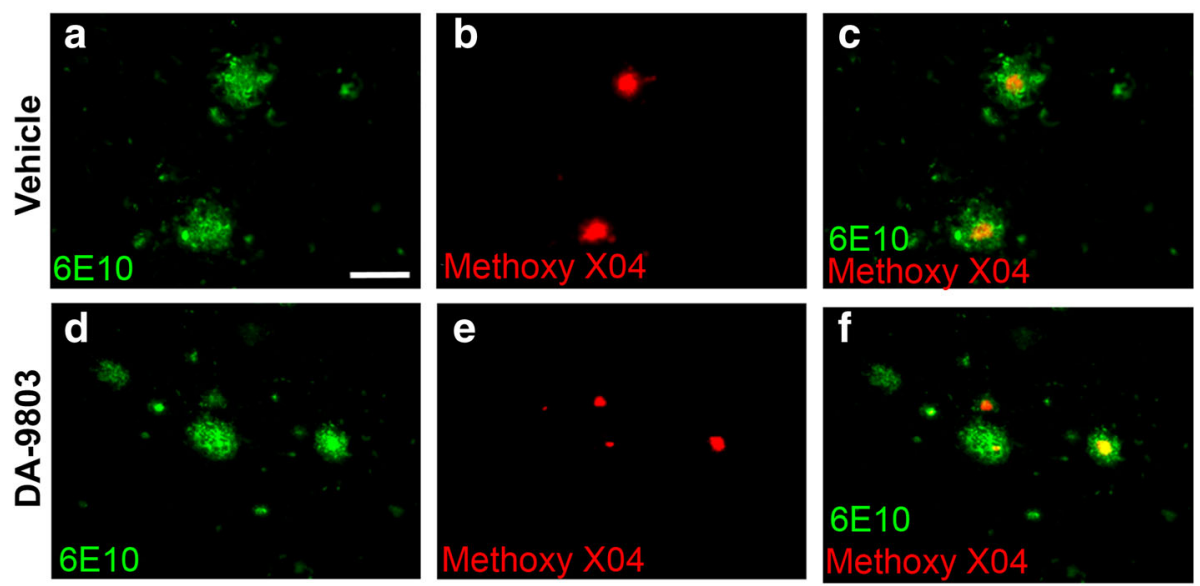

g

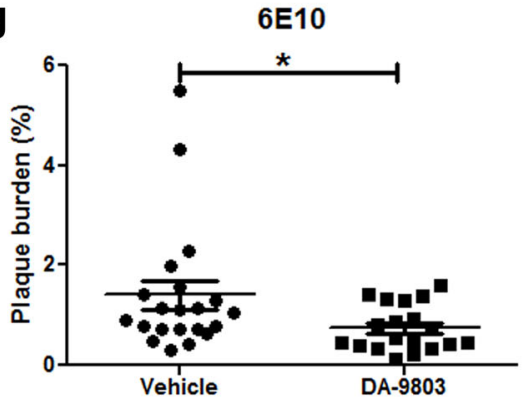

h

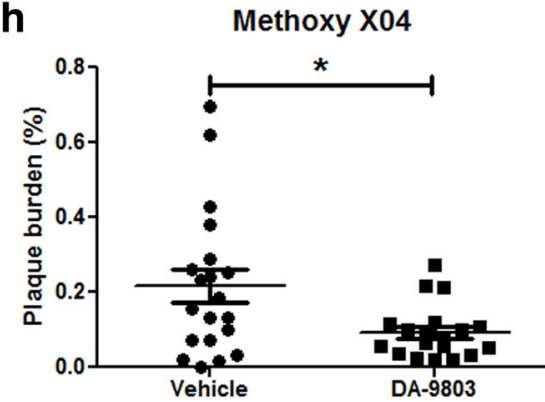

Fig. 3 DA-9803-treated mice had less postmortem cortical A 3 plaque load than vehicle-treated APP/PS1 mice. a-c Confocal microscope images of 6E10-positive (a), methoxy-XO4-labeled (b), and colabeled (c) plaques in the vehicle condition. d-f Confocal microscope images of 6E10stained (d), methoxy-XO4-labeled (e) and colabeled (f) plaques in the vehicle condition. $\mathbf{g}$ Percentage of cortex occupied by $6 \mathrm{E} 10$-positive A $\beta$ across conditions. $\mathbf{h}$ Percentage of cortex occupied by methoxy-XO4-positive plaques across conditions. Scale bar, $100 \mu m$. Mean \pm SEM. ${ }^{*} p<0.05$ 
(Additional file 2: Figure S2). These data confirm the results from the in-vivo imaging experiments.

To test whether DA-9803 or vehicle interfered chemically with methoxy-XO4 binding to plaques, fixed postmortem brain sections containing plaques were preincubated with either $10 \mathrm{mg} / \mathrm{ml}$ DA-9803, vehicle, or PBS, each followed by incubation with $4 \mathrm{mg} / \mathrm{kg}$ methoxy-XO4. The fluorescence intensity of methoxy-XO4 was comparable in all three conditions, indicating that DA-9803 did not interfere with methoxy-XO4 binding to amyloid plaques (Additional file 1: Figure S1). These data suggest that DA-9803 had a biological effect on amyloid plaque clearance rather than a direct chemical interference preventing visualization of amyloid plaques during imaging.

\section{Chronic treatment decreases the number of neurites with calcium overload in APP/PS1 mice}

Calcium homeostasis is necessary for proper neuronal function. Aberrant levels of intraneuronal calcium have been linked to altered APP processing, synaptic dysfunction, and $\mathrm{AD}$ symptoms in humans [24]. A higher proportion of neurons exhibit elevated levels of calcium (calcium overload) in aged APP/PS1 mice compared to wildtype littermates [11]. We analyzed neuronal calcium in vivo using the calcium sensor Yellow Cameleon 3.6 (YC3.6) to determine whether chronic DA-9803 treatment would restore the calcium levels to normal in neuronal processes, or neurites, exhibiting calcium overload. Elevated calcium levels or calcium overload was defined as calcium concentrations two standard deviations greater than the average calcium concentration within the neurons in wildtype mice. Thus, a neuron with YFP/CFP ratio greater than 1.79 , and a respective calcium concentration greater than $235 \mathrm{nM}$, was defined to exhibit calcium overload (Fig. 4a, yellow arrows pointing to red neurites). At baseline, prior to treatment, $6 \%$ of neurites in the drug group and $6 \%$ of neurites in the vehicle group exhibited calcium overload (Fig. 4c, d, see red boxes).

We collected additional resting calcium data during the subsequent imaging sessions, at 2, 4, and 8 weeks after treatment onset. Fluorescent angiograms were used to provide fiduciary markers to ensure the same individual neurites were identified across imaging sessions. After 2 months of treatment, the percentage of neurites with calcium overload decreased to $2 \%$ of neurites in mice treated with DA-9803 (Fig. 4b, d, e). Conversely, the percentage of overloaded neurites increased to $11 \%$ in mice treated with vehicle alone (Fig. 4a, c, e). Since the same neurites were tracked throughout the 2 months, the decrease in the number of overloaded neurites was a result of a genuine calcium restoration and not a result of neurite loss. Therefore, these data suggest that chronic treatment with DA-9803 selectively rescued overloaded neurites by restoring their baseline calcium and prevented additional neurites from exhibiting calcium overload.
Chronic treatment with DA-9803 induces morphological transformation in astrocytes and microglia in APP/PS1 mice

Activation of astrocytes and microglia has been detected in brains from AD patients and APP/PS1 mice [12, 14, 25]. To determine the effect of DA-9803 on astrogliosis, $20-\mu \mathrm{m}$ postmortem brain sections of the vehicle-treated and DA9803-treated mice were immunostained with anti-glial fibrillary acidic protein (GFAP) antibody (Fig. 5a, d, e, h) [26]. The number of GFAP-positive astrocytes in the cortex of these animals did not differ between vehicle-treated and DA-9803-treated mice (Fig. 5i). However, reactive astrocytes in both conditions were more likely to cluster around plaques, as described previously (Fig. 5i) [27]. The brain sections were also immunostained with an anti-Ibal antibody to examine microglial reactivity (Fig. 5b, d, f, h) [28]. We found no difference in Iba1-positive microglia number in DA-9803-treated and vehicle-treated animals (Fig. 5j). The number of microglia was increased in close proximity to plaques (Fig. 5j). MAP2 immunostaining revealed no obvious changes in neuronal morphology or any indication of toxicity (Additional file 2: Figure S2).

To determine the extent of astrocytic and microglial activation, we performed quantitative analysis of glial morphology. When treated with vehicle, astrocytes exhibited lengthy processes and small cell bodies (Fig. 5k-n). In contrast, DA-9803 treatment resulted in shortening of astrocytic processes and enlargement of cell bodies, consistent with activation of astrogliosis [29] (Fig. 5l-n). Similarly, DA-9803-treated microglia exhibited shortening of their processes and an increase in the cell body diameter (Fig. 5o-r), potentially suggesting a transition to a phagocytic state [30, 31].

These results suggest that DA-9803 induces activation of astrocytes and microglia, and possibly transitions glia to a state prone to $A \beta$ phagocytosis and its clearance.

\section{Discussion}

We assessed the effects of a multimodal botanical extract, DA-9803, on behavioral deficits in 5XFAD mice as well as amyloid pathology, neuronal calcium homeostasis, and neuroinflammation in APP/PS1 mice. DA-9803 is a therapeutic candidate currently in preclinical development for the treatment or prevention of $\mathrm{AD}$, with no obvious indications of toxicity. The study followed two protocols. First, 5XFAD animals underwent a 1-month gavage treatment with DA-9803 or vehicle control daily. Behavior was assessed using passive avoidance and $\mathrm{Y}$ maze paradigms. ELISAs were used to determine $A \beta 40$ and $A \beta 42$ levels in the cortex. In the second protocol, APP/PS1 animals were treated for 2 months with daily oral treatment of DA-9803 or vehicle control. Multiphoton microscopy was used to monitor plaques and intraneuronal calcium levels longitudinally in the animals. 

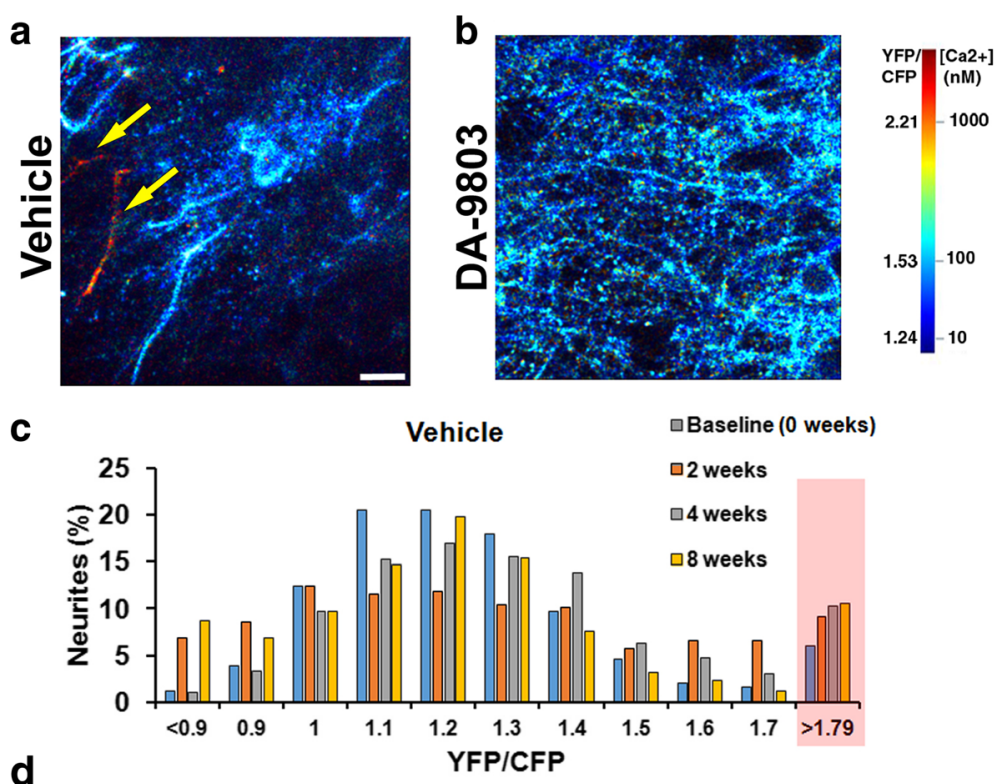

d

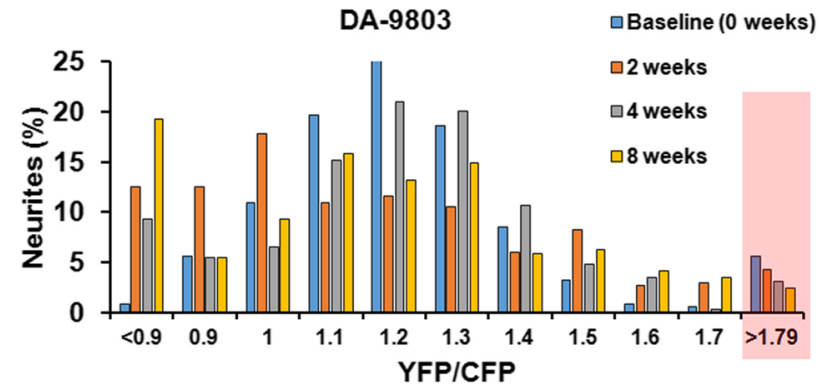

e

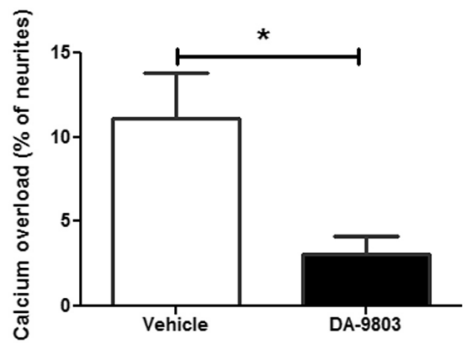

Fig. 4 Calcium overload in DA-9803-treated mice is significantly decreased after 8 weeks of treatment. a, b Two-photon images of cortical neurites, pseudocolored according to neuronal $\left[\mathrm{Ca}^{2+}\right]$ concentration and intensity, in vehicle-treated (a) and DA-9803-treated (b) mice. Red neurites exhibit elevated levels of calcium, blue neurites exhibit normal calcium levels. Yellow arrows denote overloaded neurites. c, $\mathbf{d}$ Histograms of neurite YC3.6 ratios for APP/ PS1 mice in the vehicle (c) and DA-9803 (d) conditions. $n=361$ neurites in six mice in vehicle condition. $n=290$ neurites in five mice in DA-9803 condition. e Percentage of neurites exhibiting calcium overload across conditions after 8 weeks of treatment. Scale bar, $10 \mu \mathrm{m}$. Mean \pm SEM. ${ }^{*} p<0.05$. YFP yellow fluorescent protein, CFP cyan fluorescent protein

Postmortem plaque load and neuroinflammation were also assessed. Chronic DA-9803 treatment reversed the behavioral impairments observed in 5XFAD mice. It also decreased $A \beta 40$ and $A \beta 42$ levels as well as the plaque load in the cortex. The percentage of neurites that exhibited high levels of intracellular calcium was lower in drug-treated compared to the control-treated animals. Furthermore, DA-9803 treatment led to morphological changes in astrocytes and microglia suggesting a transition to a phagocytic state.
Five-month-old 5XFAD mice were tested using the Y-maze and passive avoidance techniques after 1month $30 \mathrm{mg} / \mathrm{kg}$ and $100 \mathrm{mg} / \mathrm{kg}$ DA-9803 treatment. The animals that were treated with the vehicle alone were significantly impaired in both paradigms and had elevated levels of $A \beta 40$ and $A \beta 42$ compared to the wildtype mice. Both the $30 \mathrm{mg} / \mathrm{kg}$-treated and the $100 \mathrm{mg} / \mathrm{kg}$-treated animals performed better than their vehicle-treated counterparts on the behavioral tests. Moreover, there was no statistical 

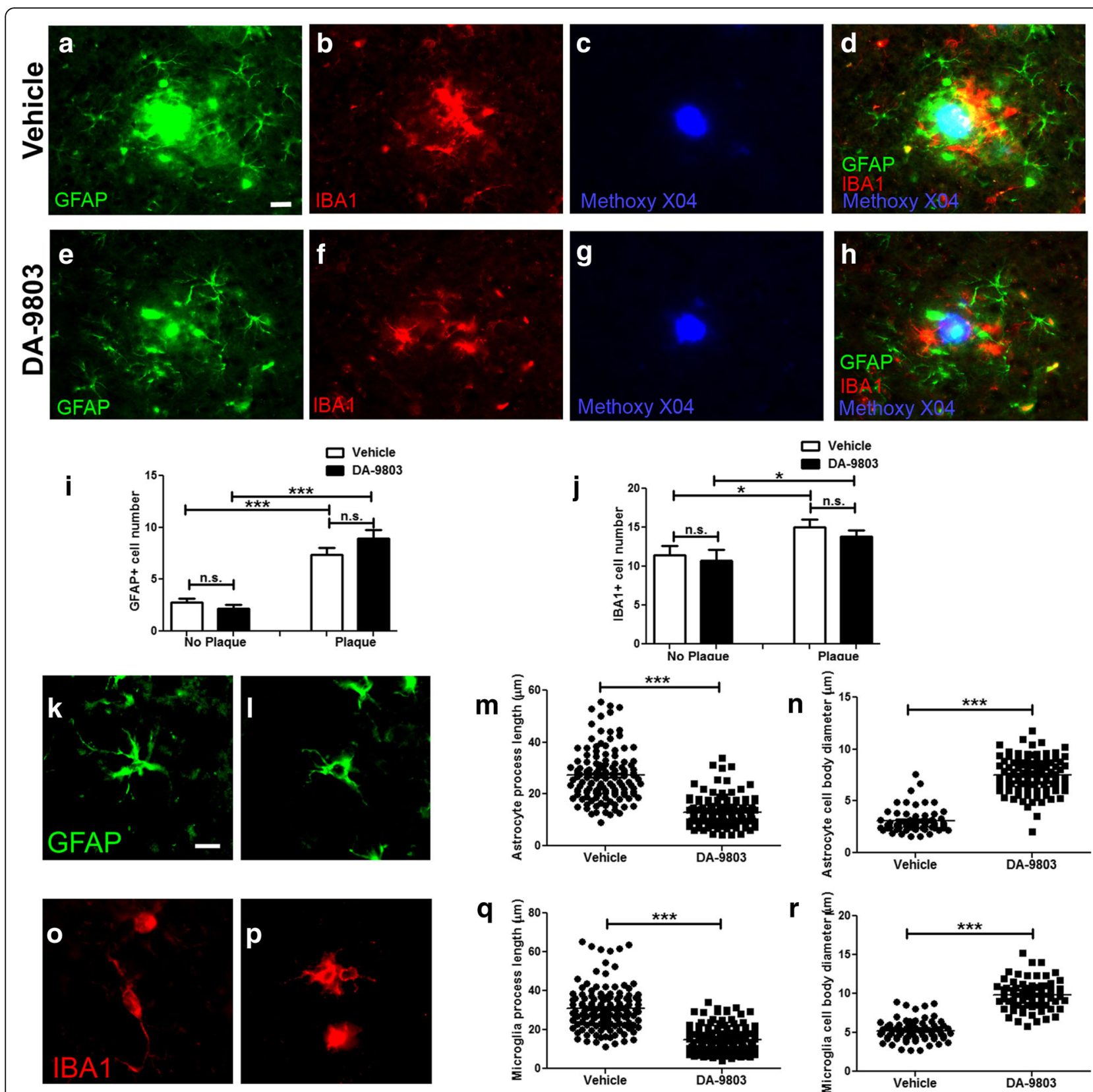

Fig. 5 IBA1 and GFAP immunoreactivity after treatment with DA-9803 and vehicle. a-d Fluorescent images of GFAP-positive astrocytes (a), IBA1-positive microglia (b), methoxy-XO4-labeled plaques (c), and colocalization of GFAP, IBA1, and methoxy-XO4 (d) in APP/PS1 mice treated with vehicle compound. e-h Fluorescent microscope images of GFAP-positive astrocytes (e), IBA1-positive microglia (f), methoxy-XO4-labeled plaques (g), and colocalization of GFAP, IBA1, and methoxy-XO4 (h) in APP/PS1 mice treated daily with $100 \mathrm{mg} / \mathrm{kg}$ DA-9803. Scale bar, 20 $\mu \mathrm{m}$. i Average number of astrocytes in close proximity (plaque) and away from plaques (no plaque) in a field of view across conditions. j Average number of microglia in close proximity (plaque) and away from plaques (no plaque) in a field of view across conditions. $\mathbf{k}$, I High-magnification fluorescent images of GFAP-positive astrocytes treated with vehicle (k) and DA-9803 (I). $\mathbf{m}$ Astrocyte process length across conditions. $\mathbf{n}$ Astrocyte cell body diameter across conditions. $\mathbf{0}, \mathbf{p}$ High magnification fluorescent images of IBA1-positive microglia treated with vehicle (o) and DA-9803 (p). q Microglial process length across conditions. $\mathbf{r}$ Microglial cell body diameter across conditions. Scale bar, $10 \mu \mathrm{m}$. Mean \pm SEM. ${ }^{*} p<0.05$, ${ }^{* * *} p<0.001$. n.s. not significant, GFAP glial fibrillary acidic protein

difference between the $30 \mathrm{mg} / \mathrm{kg}$-treated and $100 \mathrm{mg} / \mathrm{kg}$-treated animals, implying that the $30 \mathrm{mg} / \mathrm{kg}$ dose was sufficient for behavioral improvement. Interestingly, $100 \mathrm{mg} / \mathrm{kg}$ treatment reduced cortical $A \beta 40$ and $A \beta 42$ levels while $30 \mathrm{mg} / \mathrm{kg}$ treatment failed to do so. These results indicate that besides targeting $A \beta 40$ and $A \beta 42, D A-9803$ could affect additional pathways that led to improvement in the behavioral tests, supporting the multimodal nature of DA-9803. 
Individual plaques were tracked longitudinally during four separate imaging sessions throughout the 2-month drug treatment in the APP/PS1 animals. Mice treated with the vehicle alone exhibited an increase in both amyloid plaque number and burden over time, characteristic of this amyloidosis model [32]. The DA-9803-treated mice developed a limited number of new plaques in the course of the 2-month treatment compared to the vehicle-treated animals. This resulted in the DA-9803-treated animals exhibiting a lower plaque number and burden compared to the vehicle-treated mice at the end of the treatment. This result was confirmed in postmortem coronal sections of brain on a more global scale with measures of both total cortical and hippocampal amyloid plaque burden. These findings complement the immunoassay results in the 5XFAD mice exhibiting lower levels of $A \beta 40$ and A 342 after $100 \mathrm{mg} / \mathrm{kg}$ DA-9803 treatment. Taken together, we demonstrated that DA-9803 treatment rescued behavioral deficits, prevented progression of amyloid plaque deposition, and led to clearance of $A \beta$ in transgenic mouse models of AD.

The mechanisms by which DA-9803 prevented plaque deposition and/or promoted plaque clearance was unknown. DA-9803 could have inhibited $A \beta$ aggregation or synthesis. Alternatively, DA-9803 could have accelerated degradation or clearance of $A \beta$. Regardless of the exact mechanisms, targeting $A \beta$ could prevent/slow progression of $A D$, since it is likely that $A \beta$ initiates the cascade of $\mathrm{AD}$ pathology [33-35]. A $\beta$ clearance could explain the decreased plaque load in the DA-9803-treated animals. The current work, however, began with young mice exhibiting sparse plaque load, and instances of plaque clearance were rare. Future studies analyzing the effects of DA-9803 in older transgenic animals with considerable plaque load will be instrumental in testing the treatment efficacy of DA-9803 as opposed to a prevention measure for $\mathrm{AD}$ that was tested in the present study. Also, the mechanisms of action of DA-9803 will be investigated in future studies.

In addition, DA-9803's effect on calcium homeostasis was assessed in vivo. $A \beta$ has been shown to disrupt calcium homeostasis that resulted in neuronal calcium elevations [11]. Hence, restoration of intraneuronal calcium serves as an indirect functional readout of treatment efficacy. Longitudinal imaging with multiphoton microscopy allowed us to track individually identified neurites as these neurites began or ceased exhibiting calcium overload. The percentage of neurites with calcium overload in the DA9803-treated animals decreased throughout all four imaging sessions while the vehicle-treated animals continued to accumulate neurites exhibiting calcium overload during this period. The mechanism by which DA-9803 decreased calcium overload is unclear. However, it is plausible to speculate that DA-9803 neutralized the toxic $A \beta$ species, resulting in decreased $A \beta 40$ and $A \beta 42$ levels and a reduction in amyloid plaque load as described earlier, ultimately leading to decreased calcium overload. DA-9803 treatment could be affecting processes downstream of calcium homeostasis, such as restoration of calcium compartmentalization, calcineurin activity, and/or the transcriptional factor nuclear factor of activated $\mathrm{T}$ cells (NFAT) localization, all of which have been reported previously to be disrupted by elevated calcium [36].

Neuroinflammation is believed to be central to the progression of $\mathrm{AD}$. Modulating the reactivity of astrocytes and microglia could lead to increased phagocytosis and clearance of $\mathrm{A} \beta$ as well as other neurotoxic species in the CNS. This activity could lead to the prevention of amyloid deposition, increased clearance, and possible downstream effects on neural function that would include calcium overload, neuronal degeneration, and deficits in behavioral tasks. We performed immunohistochemical analyses of reactive glia in the brains of mice used in the longitudinal imaging experiments. Activation of astrocytes and microglia especially in close proximity to amyloid plaques have been reported in animal models of $\mathrm{AD}$ as well as brain tissue of $\mathrm{AD}$ patients [14, 25, 37]. Activation of astrocytes and microglia, specifically around amyloid plaques, were detected in the current study. However, there was no statistical difference in the number of either reactive astrocytes or microglia in the drug-treated animals compared to vehicle-treated mice. However, further analysis revealed morphological alterations of astrocytes and microglia after DA-9803 treatment. Shortening of the processes and increases in cell body diameter could indicate a glial transition to a phagocytic state [29-31], possibly rendering them more prone to clearing $A \beta$ by internalization of the toxic peptide [38]. This suggests that chronic treatment of DA-9807 increases a neuroinflammatory profile that may contribute to $A \beta$ clearance through phagocytosis by glial cells.

\section{Conclusions}

In summary, treatment with the botanical therapeutic DA9803 restored behavioral deficits, prevented amyloid deposition, and decreased $A \beta 40$ and $A \beta 42$ levels in the cortex. The treatment also restored calcium homeostasis and possibly facilitated neuroinflammation which could lead to enhanced clearance of $\mathrm{A} \beta$. DA-9803 is a complex extract containing multiple bioactive ingredients, and as such is likely to have multiple mechanisms of action. The current work demonstrates that DA-9803 shows promise in preventing or alleviating $\mathrm{AD}$ pathology and pathophysiology. Future studies will be directed toward characterization of the bioactive components, pharmacokinetics, potency, mechanism(s) of action, and toxicity. Considering the robust effects in the animal models, we believe that further examination of DA-9803 is warranted as a candidate therapeutic approach in $\mathrm{AD}$. 


\section{Additional files}

Additional file 1: Figure S1. Showing DA-9803 does not chemically interfere with the binding of methoxy-XO4 to amyloid plaques. A-C Fluorescent images of methoxy-XO4 amyloid plaques after preincubation with (A) DA-9803 (27 sections from one APP/PS1 mouse), (B) PBS (30 sections from one mouse), and (C) vehicle compound (29 sections from one mouse). D Fluorescence intensity of individual amyloid plaques across conditions. Scale bar, 100 um. Mean \pm SEM. (PDF 748 kb)

Additional file 2: Figure S2. Showing methoxy- $\mathrm{XO} 4$ stained amyloid plaque burden and MAP2 immunoreactivity in postmortem hippocampus after treatment with vehicle or DA-9803. A-C Fluorescent images of MAP2positive neurons (A), methoxy-XO4-labeled plaques (B), and colocalization of MAP2 and methoxy-XO4 (C) in APP/PS1 mice treated daily with the vehicle compound ( $n=14$ sections from seven mice). D-F Fluorescent images of MAP2-positive neurons (D), methoxy-XO4-labeled plaques (E), and colocalization of MAP2 and methoxy-XO4 (F) in APP/PS1 mice treated daily with $100 \mathrm{mg} / \mathrm{kg}$ DA-9803 ( $n=17$ sections from five mice). Scale bar, $100 \mu \mathrm{m}$. G Percentage of cortex occupied by methoxy-XO4-positive plaques across conditions. Mean \pm SEM. ${ }^{*} p<0.05$. (PDF $2111 \mathrm{~kb}$ )

\section{Acknowledgements}

Not applicable.

\section{Funding}

The research was funded by Dong-A ST; the Korea Drug Development Fund funded by the Ministry of Science, Information and Communication Technology; Future Planning, Ministry of Trade, Industry, and Energy, and Ministry of Health and Welfare (KDDF-201606-03, Republic of Korea); and NIH R01EB000768, R01AG044263, P30NS045776, and S10RR025645

\section{Availability of data and materials}

The datasets used and/or analyzed during the current study are available from the corresponding author on reasonable request.

\section{Authors' contributions}

GJP was involved in designing research studies, conducting experiments, acquiring data, analyzing data, and writing the manuscript. KVK was involved in designing research studies, conducting experiments, acquiring data, analyzing data, and writing the manuscript. MS was involved in designing research studies, conducting experiments, acquiring data, analyzing data, providing reagents, and writing the manuscript. SC was involved in designing research studies, conducting experiments, acquiring data, analyzing data, providing reagents, and writing the manuscript. S-hC was involved in designing research studies, conducting experiments, acquiring data, analyzing data, providing reagents, and writing the manuscript. HYS was involved in designing research studies, conducting experiments, acquiring data, analyzing data, providing reagents, and writing the manuscript. BJB was involved in designing research studies, providing reagents, and writing the manuscript. All authors read and approved the final manuscript.

\section{Ethics approval and consent to participate}

The animal experiments were conducted in compliance with Institutional Animal Care and Use Committees and $\mathrm{NIH}$ guidelines for the use of experimental animals.

\section{Consent for publication}

Not applicable.

\section{Competing interests}

MS, SC, S-hC, and HYS are full-time employees of Dong-A ST. The remaining authors declare that they have no competing interests

\section{Publisher's Note}

Springer Nature remains neutral with regard to jurisdictional claims in published maps and institutional affiliations.

\section{Author details}

Department of Neurology, MassGeneral Institute of Neurodegenerative Diseases, Massachusetts General Hospital and Harvard Medical School, 114 Sixteenth St., Charlestown, MA 02129, USA. ²Dong-A ST, Yongin-Si, Republic of Korea.

Received: 31 August 2017 Accepted: 4 January 2018

Published online: 29 January 2018

\section{References}

1. Prince $M$, et al. The global prevalence of dementia: a systematic review and metaanalysis. Alzheimers Dement. 2013;9:6-5. e2.

2. Selkoe DJ. The molecular pathology of Alzheimer's disease. Neuron. 1991; 6(4):487-98

3. Selkoe DJ, Hardy J. The amyloid hypothesis of Alzheimer's disease at 25 years. EMBO Mol Med. 2016;8(6):595-608

4. Hsiao K, et al. Correlative memory deficits, A beta elevation, and amyloid plaques in transgenic mice. Science. 1996;274(5284):98.

5. Jankowsky JL, et al. APP processing and amyloid deposition in mice haploinsufficient for presenilin 1. Neurobiol Aging. 2004;25(7):885-92.

6. Oakley $\mathrm{H}$, et al. Intraneuronal $\beta$-amyloid aggregates, neurodegeneration, and neuron loss in transgenic mice with five familial Alzheimer's disease mutations: potential factors in amyloid plaque formation. J Neurosci. 2006; 26(40):10129-40.

7. Khachaturian ZS. Diagnosis of Alzheimer's disease. Arch Neurol. 1985;42(11): 1097-105.

8. Stutzmann GE, et al. Enhanced ryanodine receptor recruitment contributes to Ca2+ disruptions in young, adult, and aged Alzheimer's disease mice. J Neurosci. 2006;26(19):5180-9.

9. Busche MA, et al. Clusters of hyperactive neurons near amyloid plaques in a mouse model of Alzheimer's disease. Science. 2008;321(5896):1686-9.

10. Bezprozvanny I, Mattson MP. Neuronal calcium mishandling and the pathogenesis of Alzheimer's disease. Trends Neurosci. 2008;31(9):454-63.

11. Kuchibhotla KV, et al. Abeta plaques lead to aberrant regulation of calcium homeostasis in vivo resulting in structural and functional disruption of neuronal networks. Neuron. 2008;59(2):214-25

12. Heneka MT, et al. Neuroinflammation in Alzheimer's disease. Lancet Neurol. 2015;14(4):388-405.

13. Cameron B, Landreth GE. Inflammation, microglia, and Alzheimer's disease. Neurobiol Dis. 2010;37(3):503-9.

14. Wyss-Coray T. Inflammation in Alzheimer disease: driving force, bystander or beneficial response? Nat Med. 2006;12(9):1005-15.

15. Kang KB, et al. Combined application of UHPLC-QTOF/MS, HPLC-ELSD and (1) H-NMR spectroscopy for quality assessment of DA-9801, a standardised Dioscorea extract. Phytochem Anal. 2017;28(3):185-94.

16. Bacskai $B$ J, et al. Imaging of amyloid- $\beta$ deposits in brains of living mice permits direct observation of clearance of plaques with immunotherapy. Nat Med. 2001:7(3):369-72

17. Kastanenka KV, et al. Optical probes for in vivo brain imaging. In: Optical Probes in Biology. CRC Press; 2015. p. 355-76.

18. Kastanenka KV, et al. Optogenetic restoration of disrupted slow oscillations halts amyloid deposition and restores calcium homeostasis in an animal model of Alzheimer's disease. PLoS One. 2017;12(1):e0170275.

19. Kastanenka KV, et al. Immunotherapy with aducanumab restores calcium homeostasis in Tg2576 mice. J Neurosci. 2016;36(50):12549-58.

20. Nagai $\mathrm{T}$, et al. Expanded dynamic range of fluorescent indicators for $\mathrm{Ca}(2+)$ by circularly permuted yellow fluorescent proteins. Proc Natl Acad Sci U S A. 2004;101(29):10554-9.

21. Levasseur JE, et al. Detailed description of a cranial window technique for acute and chronic experiments. Stroke. 1975;6(3):308-17.

22. Klunk $W E$, et al. Imaging $A \beta$ plaques in living transgenic mice with multiphoton microscopy and methoxy-X04, a systemically administered Congo red derivative. J Neuropathol Exp Neurol. 2002;61(9):797-805.

23. McCarter JF, et al. Clustering of plaques contributes to plaque growth in a mouse model of Alzheimer's disease. Acta Neuropathol. 2013;126(2):179-88.

24. Mattson MP, Chan SL. Dysregulation of cellular calcium homeostasis in Alzheimer's disease. J Mol Neurosci. 2001;17(2):205-24.

25. Akiyama $\mathrm{H}$, et al. Inflammation and Alzheimer's disease. Neurobiol Aging. 2000:21(3):383-421.

26. Eng LF, Ghirnikar RS. GFAP and astrogliosis. Brain Pathol. 1994;4(3):229-37. 
27. Pike CJ, Cummings BJ, Cotman CW. Early association of reactive astrocytes with senile plaques in Alzheimer's disease. Exp Neurol. 1995;132(2):172-9.

28. Ito $\mathrm{D}$, et al. Microglia-specific localisation of a novel calcium binding protein, Iba1. Mol Brain Res. 1998;57(1):1-9.

29. Sofroniew MV, Vinters HV. Astrocytes: biology and pathology. Acta Neuropathol. 2010;119(1):7-35.

30. Fu R, et al. Phagocytosis of microglia in the central nervous system diseases. Mol Neurobiol. 2014;49(3):1422-34.

31. Wang $Y$, et al. TREM2 lipid sensing sustains the microglial response in an Alzheimer's disease model. Cell. 2015;160(6):1061-71.

32. Garcia-Alloza M, et al. Characterization of amyloid deposition in the APPswe/PS1dE9 mouse model of Alzheimer disease. Neurobiol Dis. 2006; 24(3):516-24.

33. Hardy J, Selkoe DJ. The amyloid hypothesis of Alzheimer's disease: progress and problems on the road to therapeutics. Science. 2002;297(5580):353-6.

34. Aisen PS. The development of anti-amyloid therapy for Alzheimer's disease. CNS Drugs. 2005:19(12):989-96.

35. Lleó A, et al. Nonsteroidal anti-inflammatory drugs lower $A \beta 42$ and change presenilin 1 conformation. Nat Med. 2004;10(10):1065-6.

36. $\mathrm{Wu} \mathrm{HY}$, et al. Amyloid $\beta$ induces the morphological neurodegenerative triad of spine loss, dendritic simplification, and neuritic dystrophies through calcineurin activation. J Neurosci. 2010;30(7):2636-49.

37. Krause DL, Müller N. Neuroinflammation, microglia and implications for antiinflammatory treatment in Alzheimer's disease. Int J Alzheimers Dis. 2010;2010.

38. Shimizu E, et al. IL-4-induced selective clearance of oligomeric beta-amyloid peptide(1-42) by rat primary type 2 microglia. J Immunol. 2008;181(9):6503-13.

\section{Submit your next manuscript to BioMed Central} and we will help you at every step:

- We accept pre-submission inquiries

- Our selector tool helps you to find the most relevant journal

- We provide round the clock customer support

- Convenient online submission

- Thorough peer review

- Inclusion in PubMed and all major indexing services

- Maximum visibility for your research

Submit your manuscript at www.biomedcentral.com/submit 